\title{
Application of MET Technique after Upper Limb Dysfunc- tion after Breast Cancer Surgery
}

\author{
Huayun Liu Jingxin Wang* \\ Zhengzhou Central Hospital, Zhengzhou, Henan, 450000, China
}

\begin{abstract}
Objective: To explore the application and actual effect of MET (Muscle Energy) technology after upper limb dysfunction after breast cancer surgery. Methods: 40 women with breast cancer who underwent surgical treatment in our hospital from September 2017 to June 2019 were selected as the research object. All patients were successfully completed the modified radical mastectomy, according to different nursing methods, the patients were randomly divided into two groups, the experimental group and the control group were 20 cases respectively. Among them, the control group was given routine functional recovery exercise intervention, and the experimental group was added with met technology in the control group. One month after the operation, the functional recovery of the affected limbs of the two groups were effectively evaluated. The upper limb dysfunction of the two groups was compared by statistical method, and the ROM of shoulder joint was used for performance. Results: Through early functional recovery training and met technology, 19 ROM patients in the experimental group showed that the ROM was up to standard (95\%), and only 14 cases (70\%) in the control group were up to standard. The difference between the two groups in upper limb dysfunction was very obvious, with statistical significance $(\mathrm{P}<0.05)$. Conclusion: Early functional recovery training combined with muscle energy technology can promote the recovery of upper limb dysfunction of breast cancer patients faster and better, which is conducive to the recovery of patients as soon as possible and improve the quality of life.
\end{abstract}

\section{Keywords}

Met technology; muscle energy technology; breast cancer; upper limb dysfunction

\section{MET 技术在乳腺癌术后上肢功能障碍后应用}

\author{
刘华云 王景信 ${ }^{*}$ \\ 郑州市中心医院，中国・河南 郑州 450000
}

\section{摘 要}

目的: 探究 MET (肌肉能量) 技术在乳腺癌术后上肢功能障碍后应用和实际疗效。方法: 以我院于 2017 年 9 月一 2019 年 6 月行手术治疗的女性乳腺癌患者 40 例为研究对象, 均顺利完成乳腺癌改良根治手术, 根据不同护理方法, 将患者随机平均分 成两组, 实验组与对照组各 20 例。其中, 对照组术后给予常规功能恢复锻炼千预, 实验组在对照组基础中增加应用了 MET 技术。术后 1 个月对两组患者患肢的功能恢复状况给予有效评定, 借助统计学方式对比 2 组患者的上肢功能障碍情况, 用肩 关节活动度 ( ROM ) 进行表现。结果: 通过早期的功能恢复训练以及 MET 技术, 实验组患者中有 19例 ROM 显示达标( 95\%), 相比对照组只有 14 例达标 $(70 \%)$, 两组患者在上肢功能障碍方面的差异十分明显, 具统计学含义 $(\mathrm{P}<0.05)$ 。结论: 早 期功能恢复训练搭配肌肉能量技术能够更快、更好地推动乳腺癌患者术后上肢功能障碍的恢复, 有利于患者的尽快康复和提 高生存质量。

\section{关键词}

MET 技术; 肌肉能量技术; 乳腺癌; 上肢功能障碍

\footnotetext{
【基金项目】河南省医学科技计划项目（项目编号:

2018020778）；ＭET技术对乳腺癌术后淋巴水肿伴肩关节

活动障碍改善的临床研究（项目编号：LHGJ20191057）; JAK/STAT 信号调控巨噬细胞参与肌腱损伤后重塑形的作

用及机制研究。
}

【作者简介】刘华云（1988-）, 女, 中国河南郑州人, 初级

康复治疗师, 从事淋巴水肿、疼痛、关节活动障碍等研究。

【作者简介】王景信, 男, 现任职郑州市中心医院康复科主任。 


\section{1 引言}

乳腺癌属于女性群体中一项发病率较高的恶性肿瘤疾病。 这些年, 伴随乳腺癌的及时诊断和整体治疗的进步与提高, 病人的整体存活率和生存期获得了极大地提升, 同时乳腺癌 医治的相关合并症会干扰到病人的存活质量, 相关问题开始 逐渐引起人们的关注 ${ }^{[1]}$ 。为了进一步改善患者的存活质量, 许 多研究均指出了乳腺癌治疗的相关合并症和防治措施。其中, 以患侧上肢运动障碍较为常见, 已经发展成乳腺癌术后病人 的一项主要合并症 ${ }^{[2]}$ 。乳腺癌整体治疗的早期, 手术治疗 (包 含乳房与腋窝）以及放射治疗都容易引发局部位置瘀痕组织 的产生、纤维化和软组织的收缩, 这类情况又极易导致患者 上肢功能障碍, 引起疼痛感、活动范围缩小等, 继续发展下 去会导致肌筋膜功能障碍、黏膜性囊膜炎、神经功能受阻从 而导致疼痛和肩关节行为受阻, 上肢功能的障碍问题最终阻 碍了患者的正常生活与日常行为, 先将具体情况汇报如下。

\section{2 资料与方法}

\section{1 一般资料}

本次研究一共选择了我院于 2017 年 9 月一 2019 年 6 月 收治的 40 例行手术治疗的女性乳腺癌患者。通过随机法根据 不同护理方式, 把全部患者分成了实验组与对照组, 各 20 例 ${ }^{[3]}$ 。 根据 Richards 拟制的方案进行上肢功能障碍的确诊和划分。 具体过程是：让患者面墙而立，双手垂直上举的方式，双手 摸高水平的差异性就表示上肢上举水平存在的差异性; 安排 患者双手由背部反向进行佩戴乳罩的方式触摸自己的脊柱, 拇指所能到达的最高突出部位用于衡量上肢外展、外旋及内 旋水平。当其中任何一项出现异常, 均可判断为该侧肩关节 能力的欠缺, 即上肢功能障碍。

\section{2 篮选标准}

（1）符合标准: 乳腺癌术后患者 (术式不限)；年龄 为 29 75 岁的女性患者; 患者在知情许可同意下, 自愿加入 这次研究。

(2) 不符合标准: 存在严重的血液疾病等具备出血倾 向的患者; 存在严重的皮肤病患者; 过敏体质或存在药物过 敏史的患者; 意识不清或精神疾病患者。

\section{3 方法}

对照组病人根据中国和国际标准乳腺癌术后康复操给予
训练, 每天由专业护士定时正确引导, 包括了无阻力徒手运 动训练, 抗阻力器械运动训练、音乐一韵律康复操训练。其 中, 每项每次的训练时间是 $10 \mathrm{~min}$, 一天训练 3 次, 共半小时, 每个星期进行 2 次, 一共给予治疗 8 次。同时, 给予一定的 心理护理，对存在的护理问题进行及时、常规方式解决。

实验组患者在对照组基础上增加应用 MET 治疗, 每次 $40 \mathrm{~min}$, 一个星期做 5 次。

等长收缩后放松肌肉力量技术的主要步骤是: 病人选择 坐姿, 嘱咐其将一只手放在其背部以下, 若存在有困难则可 以把手放在同侧大转子部位, 治疗师的一只手握住病人的肘 部, 另一只手放于前臂远端部位, 尽可能拉动前臂远端脱离 背部，患者进行适当抵抗，等长收缩约为 $5 \sim 10 \mathrm{~s}$, 缓缓拉动 手臂至新的抵抗极限，反复上述动作 $3 \sim 5$ 次 ${ }^{[4]}$ 。

实施 MET 中离心性收缩肌肉力量的主要步骤是：嘱咐 患者选择卧姿，把手臂抬升至一舒适的限度，治疗师将一只 手握住胘骨远端，另一只手放于前臂位置，尽量用适当的压 力把手臂迁移超出头顶位置, 患者进行抵抗, 缓慢移动手臂 至无痛极限处, 持续 $10 \mathrm{~s}$ 左右放松, 维持于新范围内不存在 疼痛感, 若出现了疼痛需缓慢地送回。

以上动作反复进行 3 5 次, 放低手臂, 再进行该动作 $3 \sim 5$ 次。两组医疗进行的总时长为 4 个月。

\section{4 观察指标}

应用 ROM 作为检测患者上肢功能康复的客观指标。应 用专业的量角器检测健侧及患侧上肢肩关节部位于术后第 4 、 12 周肩关节活动情况（肩后伸、前屈、外展、内收四个方面）。 其中患者未产生不适或疼痛感为宜，代表上肢功能正常。

\section{5 统计方法}

使用 SPSS23.0 统计学软件对所有获取数据资料进行科 学、合理的分析与研究, 其中计数资料对比应用 $X^{2}$ 检测, 当 $P<0.05$ 时, 代表差异明显, 具统计学含义。

\section{3 结果}

通过早期功能恢复训练及 MET 技术干预，实验组中 19 例（高达 $95 \%$ 的达标率）患者显示上肢功能达标（正常）, 而对照组中只有 14 例（70\% 的达标率）患者显示达标，差 异明显, 存在统计学含义 $(\mathrm{P}<0.05)$, 具体情况如下表 1 所示。 
表 1 两组乳腺癌术后患者的肩关节活动情况对比分析（例）

\begin{tabular}{cccc}
\hline 分组 & 数量 & ROM 达标 & ROM 不达标 \\
\hline 实验组 & 20 & 19 & 1 \\
对照组 & 20 & 14 & 6 \\
\hline
\end{tabular}

注: 2 组患者的 ROM 达标率对比分析, $\mathrm{X}^{2}=3.907, \mathrm{P}=0.049$ 。

\section{4 讨论}

乳腺癌是目前危害全球女性身心健康的一项主要恶性肿 瘤, 存在着很高的发病率及致死率, 且继续呈现不断上升的 趋势。乳腺癌改良根治术是当前手术方式医治乳腺癌比较普 遍的一类临床手术，患者术后经常会产生一系列的合并症。 根据相应临床资料显示与分析, 当前乳腺癌术后出现上肢功 能障碍的概率达到了 $36 \%$ \% $5 \%$, 患侧肩关节障碍也达到了 $48 \%$, 患侧皮下积液的出现率则是 $10 \%$ 20\% 左右 ${ }^{[5]}$, 上肢水 肿回流问题通常出现在术后 1 2 年内, 其中又以上肢功能障 碍为乳腺癌术后比较普遍的一类合并症, 主要症状是患者表 现出不同程度的淋巴水肿、上肢及肩关节行为受阻、肌力降 低等功能问题。极大程度干扰患者的肢体功能, 从而影响到 患者的正常生活、社会行为。

MET 技术是一种在无痛原则下患者主动参与, 能够增加 肌肉长度、柔韧性、增强肌肉力量和稳定性的手法治疗技术。 实验组病人采用的是肌肉能量技术的方式进行治疗, 具体过 程是：等长收缩后放松（PIR），将病人的肩关节至活动的最 大程度, 并对其施加适当的阻力之后, 维持等长收缩在 $5 \mathrm{~s}$ 时 长, 对内旋肌群进行有效放松; 收缩放松 ( CR ) 过程与 PIR 基本一样, 不同地方在于需简单地在原阻力的屏蔽中完成反 复操作即可; 交互抑制（RI）; 收缩放松对抗收缩（CRAC）
方式。患者接受肌肉能量技术训练改进 ${ }^{\left[{ }^{[6]}\right.}$ 。其中, 改进肩关 节外旋的肌肉能量技术主要操作过程是, 将肩关节被动式活 动到外旋极限位置, 治疗师增加适当的阻力用于阻止病人的 内旋等长收缩, 并让该等长收缩时间持续 $5 \mathrm{~s}$, 等长收缩之后 肌群放松, 治疗师进行外旋肩关节至新的活动受阻部位, 再 次应用相同技术，在外旋的方向反复进行该技术 3 5 次。肩 关节前屈、外展、内旋过程中, 治疗师也是重复上述方式完 成肌肉能量技术的治疗过程。

综上所述，肌肉能量技术能够有效地加速乳腺癌术后上 肢功能障碍患者患侧肢体的浮肿消散, 降低疮痕挛缩的可能, 避免术后由于长时间制动和施压包扎而导致肩关节僵硬及上 肢功能障碍问题。

\section{参考文献}

[1] 刘卉. 早期功能锻炼对乳腺癌术后患肢功能恢复的效果评价 [J]. 中华全科医学, 2016,14(9):1598-1600.

[2] 沈杰, 席军府, 徐德应, 等. 肌肉能量技术配合物理疗法治疗颈型 颈椎病的疗效观察 [J]. 颈腰痛杂志 , 2016,37(5):440-441.

[3] 崔显超, 肖文武, 高少君, 等. 肌肉能量技术联合关节松动术治 疗创伤性肩周炎的疗效观察 [J]. 按摩与康复医学, 2020,11(1):37$38+42$.

[4] 覃若梅, 周薇. 乳腺癌病人术后上肢功能段炼的研究进展 [J]. 全科 护理 , 2019,17(6):675-678.

[5] 金艾香, 章小飞, 沈利凤.品管圈活动提高乳腺癌术后患者肢体功 能锻炼依从性 [J]. 当代护士, 2015(03):51-54.

[6] 蔡中博, 方桂珍. 乳腺癌术后肩关节功能障碍及其影响因素调查 研究 [J]. 护理与康复, 2015,14(12):1113-1117. 